\title{
Enseñanza de lenguas materna y extranjeras a niños con necesidades especiales: caso de Síndrome de Down ${ }^{1}$
}

\author{
Karla Ramos Rivas ${ }^{2}$ \\ Centro de Investigación Global Kids ${ }^{3}$, Costa Rica
}

\section{RESUMEN}

Durante mucho tiempo se consideró que los niños con Síndrome de Down (SD) debían aprender su lengua materna y hasta pasada la adolescencia podrían seguir con otra lengua. No obstante, los beneficios posibles del aprendizaje precoz de lenguas sobre el desarrollo cognitivo y lingüístico de estos niños han de ser reconsiderados. La exposición de niños con SD a situaciones lingüísticas distintas puede evitar ponerlo a la defensiva, lo cual tiene consecuencias favorables en lo cognitivo. lo motivacional y lo emocional. La metodología de estimulación temprana mediante los idiomas es fundamental.

\section{Abstract}

For a long time it was believed that children with Down Syndrome (DS) should learn their native language, and not until after adolescence. should they begin to study a second language. However, the potential benefits of early second-language education for cognitive and linguistic development should be reconsidered. Exposure to new linguistic situations helps children with DS to avoid defensiveness. which in turn contributes positively to the

\footnotetext{
Ponencia presentada en el I Congreso Internacional de Lingiística Aplicada. llevado a cabo en octubre de 2007, en el Campus Omar Dengo. de la Universidad Nacional de Costa Rica.

- Correo electrónico: harlu@iglobalkidscr.com

3 Global Kids es una institución dedicada a la investigación y la enseñanza de idiomas para niños. en Costa Rica: <www.globalkidscr.com>.
} 
child's cognitive, motivational and emotional level. Early stimulation with foreign languages is essential.

Palabras claves: adquisición de lengua, Síndrome de Down, aprendizaje de lengua extranjera, lingüística aplicada

Keywords: language acquisition, Down Syndrome, foreign language learning, applied linguistics

\section{Introducción}

En la actualidad, el aprendizaje y la enseñanza de lenguas extranjeras es una herramienta fundamental para los niños pues con ella actúan e influyen en el mundo globalizado. De este fenómeno no están exentos los niños con necesidades especiales y nuestros esfuerzos deberían contribuir a transformar la nuestra en una sociedad inclusiva de las diferencias. Si bien es probable que estos niños no lleguen a adquirir completamente el segundo idioma y quizás no alcancen la fluidez de un hablante nativo, es seguro que mediante la enseñanza de lenguas extranjeras una serie de dispositivos en el cerebro se estimulen y se obtengan beneficios en el nivel de la lengua materna y la extranjera.

La presente busca ser una propuesta inicial para la enseñanza de lenguas materna y extranjera a niños con Síndrome de Down (SD), con el fin de estimular el desarrollo del lenguaje a través de una metodología adaptada por Global Kids, que cuenta con un enfoque de familia. Por medio de este programa de enseñanza de idiomas procura estimular las áreas del lenguaje en el cerebro para contribuir al desarrollo de la capacidad del habla que, a su vez, tendrá repercusiones en otras áreas. Con el desarrollo actual de la investigación, se espera dar apoyo, con la información obtenida, a las familias costarricenses que.cuenten con algún niño con SD. Asimismo, se busca mejorar y extender el programa de enseñanza-aprendizaje de lenguas materna y extranjeras, pues el entorno es el encargado de potenciar habilidades o de deteriorarlas por completo. 
Ya la Historia ha documentado la discusión entre la base genética del lenguaje y las experiencias del individuo y su entorno, desde los tiempos de la Grecia antigua ${ }^{4}$. En nuestros días, se ha llegado a la conclusión de que tanto la información genética como el ambiente en el que se interactúa determinan nuestro desarrollo general y el de nuestra capacidad cognitiva: «(...) nativists wholeheartedly accept the Interactionist Thesis that cognitive characteristics are caused jointly by both internal and environmental factors» ${ }^{5}$. Si bien no podemos incidir aún en la supuesta determinación genética, lo podemos hacer modificando el ambiente externo de una persona.

Tal manipulación del medio lleva a reflexionar sobre la lingüística aplicada en el que nos compete. La noción de lingüística aplicada «alude al amplio abanico de actividades cuyo objetivo es la solución de problemas relacionados con el lenguaje o que enfrentan alguna preocupación relacionada con el mismo (...) con una metodología basada primordialmente en la lingüística (...), más la inclusión de los aportes de otras disciplinas y con un desarrollo autónomo» ${ }^{6}$. El tratamiento de nuestro caso será principalmente lingüístico con el aporte de disciplinas como la psicolingüística, la estimulación temprana, la enseñanza especial. la terapia del lenguaje, entre otras.

El tema surgió del deseo de contribuir con el cierre de brechas causadas o. más bien, ampliadas por problemas de comunicación. En Costa Rica, es un campo poco explorado por los lingüistas por lo que la actual investigación viene a ensanchar un camino que se abrió en 1983 con el estudio realizado por Bárbara Ross, e insto a otros lingüistas a realizar aportes con respecto a las poblaciones menos convencionales como lo son los adultos mayores, niños y personas con necesidades especiales. pues existe mucho por hacer aún.

\footnotetext{
4 Elizabeth Bates y otros. «Innateness and Emergentism". A Companion to Cognitive Science (Oxford: Blackwell Publishers. 1998) 590-601.

5 Richard Samuels. "Innateness in Cognitive Science», Trends in Cognitive Sciences, 8 (3), (2004): 137.

6 Francisco Adolfo Marín. "Aportaciones de la lingüística aplicada». En Vademécum para la formación de profesoress (Madrid: Sociedad General Española de Librería. 2004) 25.
} 


\section{¿Qué es el Síndrome de Down? ${ }^{7}$}

Se trata de la alteración genética más común que suele ocurrir en los seres humanos. Nuestro organismo cuenta con 46 cromosomas, repartidos en 23 pares (el óvulo tiene un miembro de cada par y el espermatozoide tiene al otro miembro), y en el caso del SD existe un cromosoma adicional en el par $21^{8}$. Se denomina «trisomía 21 estándar» y afecta el $97 \%$ de los casos.

Pero existen dos tipos más de anomalías. Uno es conocido como SD por translocación, en el cual una parte extra o una copia completa de más del cromosoma 21 se adhieren a otro cromosoma cuando el óvulo y el espermatozoide se unen para formar el embrión. La translocación ocurre en el $3 \%$ a $4 \%$ de los casos. Los sujetos con translocación tienen un déficit intelectual menor que los sujetos con trisomía estándar ${ }^{9}$. El otro tipo es el SD en mosaico. Estas personas cuentan con dos grupos distintos de células en el cuerpo: células con los 46 cromosomas típicos y células con una copia extra del cromosoma 21. Las características y los problemas comunes suelen ser más leves cuando se trata de este tipo de alteración pues no todas las células portan el cromosoma adicional. Alrededor del $1 \%$ al $2 \%$ de los casos tienen SD en mosaico.

No hay un solo gen que no intervenga en la formación y desarrollo equilibrado del cerebro; por ello, una de las mayores dificultades ven los científicos y los educadores al interactuar con personas con SD es la variabilidad entre un individuo y otro respecto del comportamiento, desarrollo, y patologías.

\footnotetext{
7 Para más información sobre el tema. ver: Patricia López y otros. «Reseña histórica del Síndrome de Down». ADM. LVII.5 (2000): 193-199: Jon F. Miller, «Language and Communication Characteristics of Children with Down Syndrome". New. Perspectives on Down S Indrome. Atlanta. 1987. 233262: y Alexandra Perovic. «Language in Down Syndrome: Delay or Principle A Effect?». Durham Working Papers in Linguistics 8 (2002): 97-110.

8 Isabel Castro. «Pasado. presente y futuro de la citogenética en Costa Rica». Rev'ista Biología Tropical 52.3 (2004): 537-544.

- Jean A. Rondal. Exceptional Language Development in Dow'n Sindrome (Melbourne. Australia: Cambridge University Press. 1995).
} 
En el Síndrome de Down existe una especificidad de origen, la presencia de un cromosoma 21 (o parte de él) extra, y ello condiciona una formación patológica en la estructura y función del cerebro, pero ésta será modulada por la expresión de los genes propios de cada persona, tanto en los presentes en el cromosoma extra como en los otros 46 cromosomas. Esta es la causa de la enorme variabilidad que se observa entre un individuo y otro con Síndrome de Down ${ }^{10}$.

En Costa Rica, la aparición de casos de SD es de entre 1:600 y 1:800 niños que nacen vivos. Esta cifra ha ido en aumento y se considera que el principal factor de riesgo es la edad de la madre. Por ejemplo:

\begin{tabular}{cccc}
\hline Edad materna & Riesgo & Edad materna & Riesgo \\
\hline 20 años & 1 en 2000 & 37 años & 1 en 250 \\
25 años & 1 en 1200 & 39 años & 1 en 150 \\
30 años & 1 en 900 & 40 años & 1 en 100 \\
35 años & 1 en 350 & 43 años & 1 en 50 \\
\hline
\end{tabular}

(Castro. El sindrome de' Down e'n el siglo xx\%. 20)7).

La posibilidad de que en una familia se dé un nuevo caso de SD dependerá del tipo de trisomía que tenga la persona afectada. Gracias a las investigaciones y a los avances en medicina y otros. la expectativa de vida hoy día para estas personas es de 60 años en Costa Rica: mientras que en 1929 era tan solo de 9 años y en 1997 de 49 años.

El SD se considera una condición y no una enfermedad. pero por las características que exhibe requiere de control médico desde el nacimiento; por ejemplo, el 50\% de los individuos requiere de alguna intervención quirúrgica. casi siempre por cardiopatía (45\%) o bien por defectos gastrointestinales $(6 \%)$. Dichas características se presentan

10 Amaia Arregui. Sindrome de Down: necesidades ceducativas y desarrollo del lenguaje (VitoriaGastiez: Gobierno Vasco. 1997) 5. 
en mayor o menor grado y se deben tomar en cuenta a la hora de preparar un programa linguístico de trabajo.

\section{Características físicas y biológicas relacionadas con la lengua}

Dos grupos de problemas deben tenerse presentes, pues afectan el proceso de adquisición de la lengua en sujetos con SD. Por un lado, existen malformaciones orgánicas y dificultades que inhiben la producción oral y la comprensión auditiva y; por otro, los procesos centrales de naturaleza cognitiva.

En primer lugar, las características más recurrentes en los aspectos biológico y físicomotor de las personas con SD que conciernen directamente la producción oral son: la hipotonía muscular en la lengua, los labios, el paladar suave y los músculos respiratorios; la cavidad bucal es muy pequeña para una lengua voluminosa y con movilidad reducida; la laringe está ubicada muy alto en el cuello y produce mucosa muy espesa; hay salivación excesiva; ocurren infecciones respiratorias, frecuentes inflamaciones de la faringe y de la laringe; suelen padecer de bronquitis que produce tos y ronquera. Otros factores que influyen en el desarrollo del lenguaje son los defectos auditivos o visuales. Por ejemplo, la pérdida de la audición es más común en las personas con SD (niños y adultos) que en personas con otra categoría de retardo mental. Además hay infecciones auditivas, estrabismo y logro lento de la coordinación binocular. El desarrollo psicomotor es tardío aunque responde bastante bien a la intervención ${ }^{11}$.

En segundo lugar, en el aspecto cognitivo se presenta la dificultad para mantener la atención; existe la experiencia sistemática del fracaso que desemboca muchas veces en enojo, limitaciones importantes en la memoria a corto plazo, problemas en el nivel de abstracción, recuperación lenta de la información en la memoria a largo plazo, entre muchas otras manifestaciones que perturban la adquisición y el

II Arregui. 
desarrollo del lenguaje. En el cerebro existe una reducción importante en el número de neuronas, lo cual afecta el funcionamiento de ciertas partes del mismo y en consecuencia hay un retraso mental (entre leve y severo) que marca la diferencia entre la edad mental y la edad cronológica de la persona. Sin embargo, debe tomarse en cuenta que no sólo la edad cronológica y la edad mental discrepan, sino también la edad del lenguaje expresivo, la edad del lenguaje receptivo, la edad lingüística compuesta y la edad social ${ }^{12}$.

Estudios anteriores de Moor (1967) y Gibson (1981), citados por Rondal ${ }^{13}$, señalan que en el nivel cognitivo, el cociente intelectual para la trisomía 21 estándar es entre los 45 y los 50 puntos. Asimismo, se considera que el crecimiento mental de estas personas culmina entre los 10 y los 15 años de edad cronológica para una correspondencia con la edad mental de una persona de 5 años aproximadamente. «One can wonder how language can develop at all in such adverse conditions. The fact that it does to some extent in most DS individuals may certainly be taken to indicate the robustness of basic language organization in humans» ${ }^{14}$.

Desde el punto de vista neurobiológico, Fidler indica que las diferencias entre el cerebro de un niño con desarrollo normal del de un niño con SD son imperceptibles cuando el feto aún está en el vientre $^{15}$. No es sino hasta que el niño alcanza los 6 meses de edad después de nacido que las diferencias son más determinantes.

Aunque se presentan distintos grados de impedimentos, tanto en las habilidades cognitivas como linguísticas, los investigadores coinciden en que las personas con SD tienen una restricción significativamente mayor en el desarrollo de su habilidad lingüística. No

12 Gilberto Cascante. El trastorno especifico del lenguaje: factores que afectan la adquisición y el desarrollo del lenguaje en niñosde 3 a 6 años (San José: GICLA. 2007).

1: Rondal. 5.

it Rondal. 5.

15 Deborah J. Fidler y otros, «Education and Children with Down Syndrome: Neuroscience. Development, and Intervention». Mental Retardation and Dev'elopmental Disabilities Research Reviev's, 13 (2007): 262-271. 
obstante, parece no existir «consenso en cuanto a la naturaleza del déficit linguístico» ${ }^{16}$ por la gran cantidad de factores que entran en juego. A pesar de su barrera con el lenguaje oral, los individuos con SD cuentan con una fortaleza en la memoria visuo-espacial, de ahí que puedan demostrar competencia en algunos aspectos de la lectura y en particular, la identificación de palabras. Lo interesante es que hay evidencia de que los niños con SD recurren a sus habilidades visuo-espaciales y verbales cuando deben leer.

Los niños con SD han demostrado adquirir conceptos básicos de objetos mucho más lento que un niño con desarrollo normal; no obstante, también se ha demostrado que con una intervención temprana y extensa ellos logran adquirir los conceptos en un tiempo muy parecido $^{17}$. Pese a ello, un problema emerge en este estadio y es la inestabilidad de la adquisición. El desempeño después de haber adquirido algún concepto es extremadamente variable y aparentemente está relacionado con dificultades emocionales; es decir, gracias a la intervención nuevas habilidades pueden aparecer pero desaparecen poco tiempo después. Esto se debe al impedimento que esta población enfrenta con la memoria a corto plazo, cuyo origen se halla en la disfunción del hipocampo. Estos problemas de aprendizaje y de memoria emergen en la infancia tardía y se vuelven considerablemente más notables conforme el niño crece y, ahí radica la importancia en que las intervenciones se realicen en la infancia temprana.

\section{Limitaciones lingüísticas específicas}

Rondal agrupa las limitaciones lingüísticas en cinco categorías: fonética y fonológica, léxica, estructuras semánticas, morfosintáctica y pragmática. En cuanto a los problemas fonéticos y fonológicos, se presentan dificultades para la producción de sonidos específicos

16 Marina Tsakiridou, «The Linguistic Profile of Down`s Syndrome Subjects: Evidence from WhMovement Construction», SOAS Working Papers in Linguistics, 14. 2006: 229.

17 Fidler. 
como los fricativos; mientras que los sonidos vocálicos, semivocálicos, nasales y oclusivos son adquiridos de primero.

El léxico es reducido, aunque utilizado de manera correcta y resulta comprensible en la mayoría de los casos. Por lo general, el vocabulario comienza a ampliarse a partir de los cuatro años de edad cronológica. Con respecto a las estructuras semánticas, éstas muestran un amplio rango de significados relacionales tales como los reportados para el típico proceso de adquisición del lenguaje; cito:

Examples of early semantic relations expressed by DS (...) are 'notice or existence', 'disappearance', 'recurrence', 'attribution', 'possession', 'location', 'agent-action', 'action-patient', 'agentaction-patient' ${ }^{18}$.

La morfosintaxis, por su parte, se caracteriza por contar con poca elaboración de los elementos morfológicos y sintácticos en elocuciones telegráficas cortas. Suele haber omisión de palabras función como los artículos. auxiliares, cópulas, pronombres, conjunciones, preposiciones, y de elementos morfológicos como la inflexión verbal o nominal. El desarrollo gramatical no llega a ser completo en sujetos con SD aunque el orden sintáctico, por lo general, es correcto. Se ha explicado esta carencia gramatical al considerar que los niños con SD son más sensibles a los sonidos al inicio de las palabras que al final de éstas ${ }^{19}$.

Finalmente. se ha mostrado que la pragmática es el aspecto menos afectado del lenguaje, pero sí se encuentran barreras con respecto a las anáforas y a los pronombres. Las personas con SD cuentan con un nivel de lengua informativo y de intercambio de información

is J. A. Rondal. Exceptional Lunguage Development in Down Sỵdrome (Melbourne. Australia: Cam hridge University Press. 1995) 7.

11) Margriet A. Groen y otros. "A Case of Exceptional Reading Accuracy in a Child with Dow'n Sy" drome: Underlying Shills and the Relation to Reading Comprehension". Cognitive Neuropsyc/lulı" g. 23.8 (2006): 1190-1214. 
nueva. El cambio de turnos en la conversación suele ser respetado. Estas persones pueden utilizar la mayoría de los tipos de oraciones como declarativas, imperativas e interrogativas. Una dificultad que enfrentan en este aspecto es la toma de decisiones una vez que las demandas cognitivas sociales aumentan:

(...) as the demands and complexities of social situations increase in middle childhood and beyond, individuals with Down Syndrome may show difficulties with social strategies, especially as they enter adolescence and face changes in emotional functioning and $\operatorname{mood}^{20}$.

\section{El retraso mental en personas con SD}

Se solía pensar que los niños con SD se caracterizaban por un simple retraso en el proceso de adquisición del lenguaje, aunque seguían el mismo patrón de desarrollo del de un niño normal pero con una demora evidente ${ }^{21}$. A esto se le conoce como la Hipótesis del Retraso de corte cuantitativo, pues mide el tiempo en que aparecen ciertas producciones orales. Hoy día, los estudios han criticado esta posición según nuevos resultados y se ha propuesto la Hipótesis de la Diferencia de corte cualitativo; es decir. ésta se centra en las incompatibilidades que existen entre las producciones orales de una persona con desarrollo normal de otra con $\mathrm{SD}^{22}$. En relación con las habilidades de comprensión de lectura, la memoria a corto plazo y la gramática se destacan como dos áreas que representan alguna dificultad para los individuos con $\mathrm{SD}^{23}$.

Se conocen casos extraordinarios de personas con SD que son funcionales en la sociedad, algunas de ellas han realizado estudios

\footnotetext{
3) Fidler. 267.

21 Artegui.

$\because$ Tsakiridou.

$\because$ Groen.
} 
universitarios y fungen como profesores, actores, etc. Todos estos casos están relacionados con un desarrollo lingüístico cuasi-normal. Un caso excepcional de desarrollo lingüístico fue estudiado por Jean A. Rondal entre los años 1988 y 1992 en la ciudad de Liège, Bélgica. El sujeto de estudio era Françoise, con 32 años de edad cronológica al momento del inicio de las pruebas y con 36 años al momento de terminar las pruebas. Su cociente intelectual rondó siempre los 65 puntos. Su edad mental verbal era de 9 años y 10 meses y una edad mental no verbal de 5 años y 8 meses, para una edad mental compuesta de 7 años y 4 meses. La pregunta principal del estudio apuntaba a si el desarrollo y el funcionamiento normal o cuasi-normal de la lengua eran posibles a pesar de las limitaciones cognitivas centrales, particularmente las conceptuales. A esto se respondió que sí, aunque de manera parcial. El funcionamiento fonológico y gramatical es posible porque ambos aspectos de la lengua son independientes de otros procesos centrales.

Los resultados de este estudio favorecen la hipótesis de un débil ligamen entre la cognición y los componentes particulares de la lengua tales como la fonología y la sintaxis. Por lo tanto, se arguye que los casos de desarrollo lingüístico excepcional como Françoise. operan sobre la base del conocimiento de una gramática implícita que los individuos no pueden desarrollar a través del aprendizaje o el crecimiento conceptual dadas sus limitaciones cognitivas. Que este conocimiento resulte innato o no, es de poca importancia ya que de igual manera se alcanzan mejoras significativas gracias a la intervención temprana en los niños; lo que importa es potenciar en ellos sus habilidades en el momento adecuado y de la manera adecuada.

\section{¿Cuáles son los beneficios de adquirir una lengua extranjera?}

Para Cenoz, los estudios de plurilingüismo tanto en el medio natural como escolar apuntan a que la adquisición de más de dos lenguas es posible y no presenta problemas en el desarrollo cognitivo 
de una persona ${ }^{24}$. Más bien, Cenoz se inclina a pensar que el bilingüismo puede desarrollar la conciencia metalinguística y la creatividad de las personas. En los niños con Síndrome de Down esto no ha sido probado, aunque se puede hablar con mayor seguridad de otros beneficios. Si quisiéramos mencionar una ventaja de los hablantes multilingües sobre los monolingües tendría que ser la conciencia metalingüística que se desarrolla puesto que la mayoría de los autores concuerdan con esto. La definición de este término que Correa toma es:

Metalinguistic awareness is the ability to think flexibly and abstractly about language; it refers to an awareness of the formal linguistic features of language and the ability to reflect thereupon (...). To be metalinguistically aware, then, is to know how to approach and solve certain problems which themselves demand certain cognitive and linguistic skills ${ }^{25}$.

Otros beneficios al adquirir una segunda lengua se derivarían de tener que negociar el significado de las palabras entre los interlocutores echando mano del contexto, los gestos, las marcas de entonación, la mirada y otros aspectos linguiísticos y extralingüísticos. Además, otros beneficios se derivarían de la elección de tareas motivadoras para provocar en el alumno el sentimiento de éxito y el deseo de comunicar.

En el nivel emocional y motivacional, el sentimiento de éxito que supone adquirir una segunda lengua o adquirir una competencia lingüística en su lengua materna aumenta la autoestima y, consiguientemente, los niños crean una actitud favorable hacia esta lengua porque les proporciona experiencias positivas que quizás no hayan tenido antes. En el nivel cognitivo y lingüístico, el aprendizaje de

\footnotetext{
I4 J. Cenoz, «Universitat de Barcelona». 2003. 23 de Febrero de 2007 <http://www.ub.es/ice/portaling/eduling/cat/n_._/cenoz-article-nl.pdf $>$.

25 M. Correa, Literacy; Metalinguistic Aw'areness and Multi-Competence (2004). Recuperado el 21 de agosto de 2007. de Center for Computing and Information Technology. University of Arizona. $<$ http://www.u.arizona.edu/ maite/academic/ papers/metalinguistic.pdf>. p. 5.
} 
lenguas extranjeras permite desarrollar una reflexión acerca del lenguaje $y$, en particular, acerca de la lengua materna. Aprender a comunicarse es poner en marcha estrategias de comunicación y de aprendizaje útiles en diversos campos ${ }^{26}$. Por ejemplo, se ha confirmado el desarrollo de una capacidad para la resolución de problemas gracias al conocimiento de dos o más lenguas. Con la ayuda del contexto (lugar, situación, gestos, mirada, entonación, y otros), los niños no sólo logran mayor competencia en la segunda lengua sino que a la vez se contribuye a desarrollar la competencia linguiística en su lengua materna.

\section{Recomendaciones}

Todo programa de enseñanza dirigida a este grupo debe contemplar el desarrollo físico y motor, porque la estimulación física favorece el desarrollo del lenguaje, desde el punto de vista de la comprensión y de la producción. Por eso es esencial el uso de gestos o señas que acompañen la lengua oral para promover la comunicación alternativa.

La enseñanza temprana debe ser individualizada, para garantizar una mayor eficacia. La estimulación temprana se recomienda para aprovechar la plasticidad neuronal propia de la niñez. Con la edad, esta plasticidad se va perdiendo, lo que implica un esfuerzo mayor para adquirir nuevos conocimientos. Esta estimulación temprana mediante

\footnotetext{
30 Otras referencias. para profundizar en el tema: Mercedes Belinchón y otros. Psicologia del lenguaje. Investigación y teoría (Madrid: Trotta. 1992): Monica Beyer, Baby Talk: A (juide' to Using Basic Sign Language to Communicate with Your Baby (Nueva York: Penguin Group. 2()(1)6): Dorothy V. M. Bishop, «Cognition: Enhanced: An Innate Basis for Language?», Science 286.5448 (1999): 2283-2284: Noam Chomsky. Lu arquitectura del lenguaje (Barcelona: Kairós. 200)()) y Réflexiones acerca del lenguaje (México: Trillas. 1981): Stephen Crain y Paul Pictroski. «Nature, Nurture and Universal Grammar». Linguistics and Philosoplyy 24 (2001): 139-185: Glenn Doman y otros, Cómo multiplicar la inteligencia de su bebé: la revolución pacífica (Madrid: EDAF. 1999): Miguel Serra, La adquisición del lenguaje (Barcelona: Ariel, 2000): Orlando Terré. Néurodésarrollo infantil y estimulación temprana (San José: Santa Paula. 2007), y Transtomos y alteraciones en el lenguaje infantil (San José: Santa Paula. 2007): Elisabeth Tucker y otros. «Using a Three-Step Decoding Strategy with Constant Time Delay to Teach Word Reading to Students with Mild and Moderate Mental Retardation». Focus on Autism and other Developmental Disabilities 2.32 (2008): 67-78.
} 
las lenguas puede hacerse en conjunto con los padres de familia, como primera etapa y posteriormente, pasar a una etapa más independiente y luego a otra más social. Una vez que el niño haya alcanzado seguridad y autoestima deberá ir socializando también con personas de su edad para evitar el aislamiento.

Con el fin de reafirmar su autoestima e independencia, el profesor o padre de familia nunca debe hacer las tareas o actividades por el niño; se le deben dar las herramientas y el tiempo necesario para que él logre por sus propios medios lo que se le pide. De esta manera lograremos demostrarle su autonomía para hacer las cosas.

Para la enseñanza de lenguas, el programa debe estar centrado en lenguaje funcional; es decir, el niño debe aprender a saludar, a disculparse, a responder preguntas, a expresar sus sentimientos antes que aprender otras cosas. De esta manera se lo estará preparando para ser parte del mundo y no experimentará un sentimiento de fracaso si sabeconducirse de acuerdocon ciertas reglas sociales. El léxico, trabajado por campos semánticos, también deberá ser constantemente alimentado y refrescado para evitar sentimientos de enojo o frustración.

El léxico debe ser reforzado no sólo en el nivel oral sino también en el nivel visual. Vivimos en una sociedad donde el código escrito y simbólico tiene mucho peso; por lo tanto, una vez que el niño ya conoce diez palabras, las podremos escribir con letra grande y nítida en cartulina y acompañar las ilustraciones con las palabras que las designan. No sólo estimulamos el desarrollo de la capacidad del habla sino también la capacidad de lectoescritura. En términos de sintaxis, morfología y pragmática se deberá ir trabajando paulatinamente una vez que se haya alcanzado el dominio de 200 palabras.

En la clase de idiomas, cualquier logro o esfuerzo debe ser reconocido. Para que existan estos logros, al niño deben recordársele las actividades exitosas una y otra vez para que pueda irse desvinculando del contexto y pueda dar significado a las palabras sin recurrir a elementos extralinguiísticos. Así alcanzará una buena competencia lingüística y una competencia comunicativa: «un proceso que nos 206 
lleva toda la vida, no puede ser un proceso fácil de construir y desarrollar ${ }^{27}$. Será decisivo contar con un personal altamente capacitado y muy motivado, que realmente crea en el potencial de los niños, para lograr su mejor esfuerzo.

Nuestra investigación ya cuenta con un año y medio de desarrollo y ejecución. El estudio se inició con Ana Lucía Soto ${ }^{28}$ el 6 de agosto de 2007, cuando tenía cinco años recién cumplidos. Debido al avance experimentado con ella, se comenzó a extender la aplicación a otros niños. En julio de 2008, ingresaron al centro tres niños más y en el corto plazo avances significativos con todos ellos.

Los logros inmediatos del programa son: el deseo que se les despierta por empezar a comunicarse e interactuar con otros, el desarrollo de su capacidad para realizar tareas de asocie y la tolerancia a las mezclas de letras, el desarrollo de la capacidad para la identificación de palabras, el desarrollo para la capacidad de producción de sonidos y producción de palabras, aprendizaje de vocabulario a través de señas lo que permite una comunicación alternativa, reducción de la frustración tanto en el niño como en sus familiares, aprendizaje de estrategias para resolver problemas, entre otros.

Aunque la educación bilingüe de niños con SD va en aumento. es aún relativamente poco lo que se ha escrito al respecto y los miedos siguen atormentando a profesionales y padres de familia. A los lingüistas en Costa Rica les falta mucho por recorrer.

\footnotetext{
Cascante, 64.

Ana Lucía (Lucy) Soto tiene seis años actualmente y vive en Heredia. Costa Rica. Ingresó a Global Kids con el fin de estimular su desarrollo en español. su lengua materna. Gracias a ella. pudimos poner en marcha nuestro proyecto de enseñanza y lo hemos podido adaptar $y$ ' mejorar.
} 